\title{
Watch Out for the Unexpected: Sole Gallbladder Metastasis in a Patient with Malignant Melanoma Striked by FDG-PET
}

\author{
Okuyucu Kursat ${ }^{1}$, Alagoz Engin ${ }^{1}$, Arslan Nuri ${ }^{{ }^{*}}$, Komurcu Seref ${ }^{2}$, Ayan Aslı ${ }^{1}$ and Ozturk Erkan ${ }^{3}$ \\ ${ }^{1}$ Department of Nuclear Medicine, Gulhane Military Medical Academy and Medical Faculty, Ankara, Turkey \\ ${ }^{2}$ Department of Medical Oncology, Gulhane Military Medical Academy and Medical Faculty, Ankara, Turkey \\ ${ }^{3}$ Department of General Surgery, Gulhane Military Medical Academy and Medical Faculty, Ankara, Turkey
}

*Corresponding author: Nuri Arslan, Department of Nuclear Medicine, Gulhane Military Medical Academy and Medical Faculty, Ankara, Turkey, Tel: + 903123044801; E-mail: narslan@gata.edu.tr

Received date: Dec 31, 2014, Accepted date: Jan 31, 2015, Publication date: Feb 03, 2015

Copyright: (C) 2015 Kursat O, et al. This is an open-access article distributed under the terms of the Creative Commons Attribution License, which permits unrestricted use, distribution, and reproduction in any medium, provided the original author and source are credited.

\begin{abstract}
Metastatic gallbladder disease is a rare pathology. Gallbladder metastasis of malignant melanoma is especially rarer. Herein we present the solitary metastasis of a malignant melanoma to the gallbladder.

47-year old male having an ulcerated skin lesion at right toe was diagnosed as malignant melanoma with excisional biopsy. Thereon the patient without locoregional disease was treated with large surgical resection. A metastatic inguinal lymph node was excised 4 months later and interferon-alpha treatment was begun. FDG-PET imaging was requested to evaluate the extent of disease after 15 days and any metastatic focus was not detected.
\end{abstract}

A control FDG-PET was performed for the evaluation of therapy 8 months later. There was only an unexpected, markedly increased focal FDG uptake arousing doubt in the gallbladder fossa on the whole-body images. This accumulation was proven histopathologically as malignant melanoma metastasis after exploratory cholecystectomy and the patient was treated by dacarbazine+cisplatin.

Physicians interpreting FDG-PET scans of patients with malignant melanoma must be cautious about the significance of prominent unusual uptakes at unexpected localizations. It is mandatory to establish a certain histopathologic diagnosis if possible.

Keywords: Gallbladder metastasis; Malignant melanoma; 18 fluoro deoxy-glucose positron emission tomography (FDG-PET)

\section{Introduction}

Malignant melanomas have the potential of metastasizing to any organ in the body. The most frequent distant metastatic localizations are lung, liver, skin and gastrointestinal tract [1]. In gastrointestinal tract, the most common metastatic site is small intestine [2]. Gallbladder metastasis of malignant melanoma is rare, isolated solitary one is even extremely rarer. Gallbladder lesions often occur accompanying widespread disease and generally stems from skin melanoma [3]. But interestingly malignant melanoma is the leader of all metastatic gallbadder tumours accounting for $50-60 \%$ of all cases [4].

It is being widely benefited from FDG-PET for malignant melanoma patients in the primary staging, evaluating treatment response and restaging [5]. In routine patient management, localized disease (without locoregional and distant metastasis) is treated surgically and then followed by FDG-PET. If there is metastasis on control FDG-PET, appropriate systemic therapy algoritm is applied $[6]$.

\section{Patient Findings}

47-year old male having an ulcerated skin lesion at right toe was diagnosed as malignant melanoma with excisional biopsy (Breslow: 1.5 $\mathrm{mm}$, Clark level IV). Thereon the patient without locoregional disease (sentinel lymph node negative, stage II) was treated with large surgical resection. 4 months later a metastatic inguinal lymph node seen on computed tomography (CT) was excised and interferon-alpha treatment was begun. FDG-PET imaging was requested to evaluate the extent of disease after 15 days and any metastatic focus was not found.

A control FDG-PET was performed for the evaluation of interferon-alpha therapy 8 months later. Although any certain pathologic accumulation consistent with locoregional or distant metastasis was not detected on whole-body screening, there was an unexpected, markedly increased focal FDG uptake in the gallbladder fossa (Figure 1).

This activity was corresponding to the area of gallbladder wall thickening and nodularity extending into the lumen on CT with oral and IV contrast (Figure 2A). The patient subsequently underwent abdominal ultrasonography (USG) and a soft tissue mass of 30x23 mm was identified in the gallbladder (Figure 2B). 


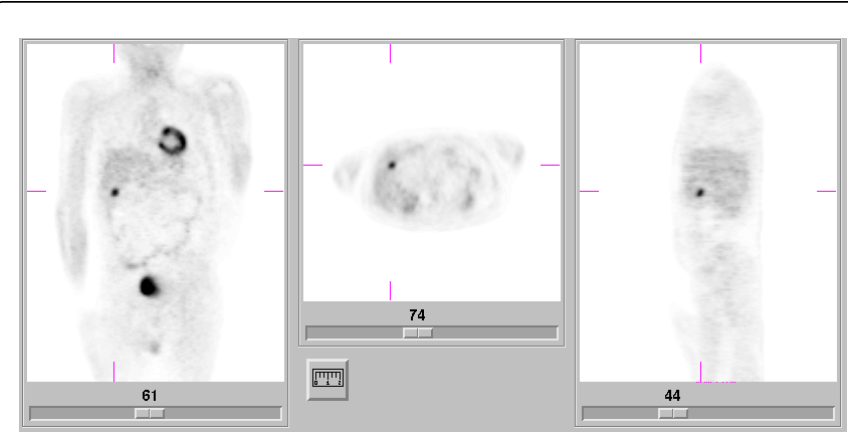

Figure 1: Coronal (A), transaxial (B) and sagittal (C) FDG PET slices showed marked focal increased FDG uptake in the gallbladder corresponding to the mass seen on CT and US.

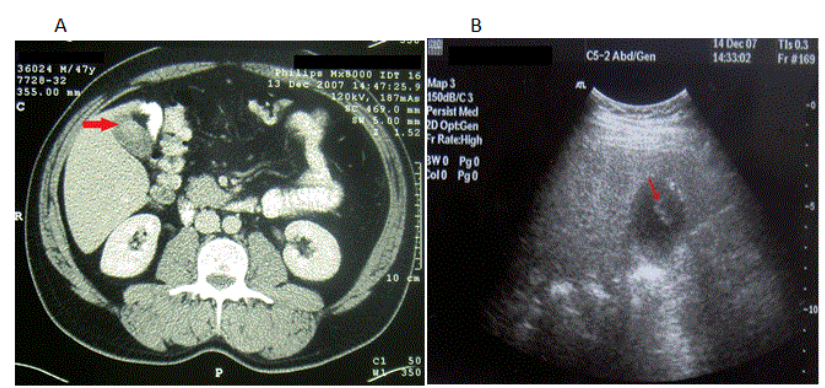

Figure 2: A soft tissue mass in the gallbladder (arrow), 30x23 $\mathrm{mm}$ in size, was detected on abdominal CT (A) corresponding to the mass (arrow) seen on US (B).

A benign gallbladder pathology was thought according to the clinical and conventional radiological findings at first sight. As exploratory laparotomy/ cholecystectomy was performed for confirmation of the lesion, histopathological examination revealed a malignant melanoma metastasis and 9 metastatic lymph nodes. Whereupon dacarbazine+cisplatin combination was added to the therapy regimen.

\section{Discussion}

Sudden emerging of an atypical FDG accumulation in the follow-up of cancer patients creates a challenge that must be illuminated for the clinician. Isolated solitary gallbladder metastasis can be confused with primary gallbladder pathologies (gallbladder cancer, acute cholecystitis), especially if the patient is in disease-free interval for a long time. This case demonstrates a striking example about the importance of any incidental focal FDG uptake which might represent an unexpected finding such as a malignant melanoma metastasis. FDG-PET imaging has excellent sensitivity for metastatic melanoma tending to be a high-grade, aggressive tumour characterized by its high metabolic activity resulting in prominent FDG avidity [7]. Thanks to this pecularity, FDG-PET can easily detect unexpected metastasis at unusual places hardly evaluated by conventional imaging methods (US,CT,MRI). So it is a useful technique in correct staging and management of patients with melanoma. CT and US just give confined anatomical information not detailing the disease activity [8]. FDGPET is more sensitive than CT and US for detection of malignant melanoma metastasis and increases the accuracy of staging. It also changes therapy protocol when locoregional and/or distant metastasis are detected. This condition necessitates implementation of new chemotherapeutic agents. If there is only gallbladder uptake strongly implying metastasis in malignant melanoma, a simple cholecystectomy plus chemotherapy is enough in favour of the patient as is in our case.

\section{Conclusion}

Physicians interpreting FDG-PET scans of patients with malignant melanoma must be cautious about the significance of prominent unusual uptakes at unexpected localizations and correlate them with conventional radiological images and clinical findings. Any markedly increased focal FDG accumulation arousing doubt should be followed up meticulously. If and whenever possible, it is mandatory to establish a certain histopathologic diagnosis.

\section{References}

1. Balch CM, Houghton AM (1992) Diagnosis of metastatic melanoma at distant sites. Cutaneous melanoma (2ndedn.) Lippincott, Philadelphia, 439-467.

2. Ollila DW, Essner R, Wanek LA, Morton DL (1996) Surgical resection for melanoma metastatic to the gastrointestinal tract. Arch Surg 131: 975-979.

3. Furumoto K, Miyauchi Y, Ito D, Kitai T, Kogire M (2013) Solitary metastatic gallbladder malignant melanoma originated from the nasal cavity: A case report. Int J Surg Case Rep 4: 965-968.

4. Blecker D, Abraham S, Furth EE, Kochman ML (1999) Melanoma in the gastrointestinal tract. Am J Gastroenterol 94: 3427-3433.

5. Basu S, Houseni M, Alavi A (2009) (18)F-FDG-PET in restaging primary maxillary sinus melanoma with isolated gall bladder metastasis. Hell J Nucl Med 12: 170.

6. Danielsen M, Højgaard L, Kjær A, Fischer BM (2013) Positron emission tomography in the follow-up of cutaneous malignant melanoma patients: a systematic review. Am J Nucl Med Mol Imaging 4: 17-28.

7. Rehani B, Strohmeyer P, Jacobs M, Mantil J (2006) Gallbladder metastasis from malignant melanoma: diagnosis with FDG PET/CT. Clin Nucl Med 31: 812-813.

8. Holloway BJ, King DM (1997) Ultrasound diagnosis of metastatic melanoma of the gallbladder. Br J Radiol 70: 1122-1125. 